\title{
Wireless Sensor Network Dynamic Mathematics Modeling and Node Localization
}

\author{
Xiaoyang Liu $\mathbb{D}^{1,2}$ and Chao Liu ${ }^{1,3}$ \\ ${ }^{1}$ School of Computer Science and Engineering, Chongqing University of Technology, Chongqing, 400054, China \\ ${ }^{2}$ College of Engineering, The University of Alabama, Tuscaloosa, Alabama, 35401, USA \\ ${ }^{3}$ College of Automation, Chongqing University, Chongqing, 400044, China
}

Correspondence should be addressed to Xiaoyang Liu; lxy3103@163.com

Received 13 November 2017; Revised 13 April 2018; Accepted 2 May 2018; Published 31 May 2018

Academic Editor: Maode Ma

Copyright (C) 2018 Xiaoyang Liu and Chao Liu. This is an open access article distributed under the Creative Commons Attribution License, which permits unrestricted use, distribution, and reproduction in any medium, provided the original work is properly cited.

\begin{abstract}
With the rapid development of wireless sensor network (WSN) technology and its localization method, localization is one of the basic services for data collection in WSN. The localization accuracy often depends on the accuracy of distance estimation. Because of the constraint in size, power, and cost of sensor nodes, the investigation of efficient location algorithms which satisfy the basic accuracy requirement for WSN meets new challenges. This paper proposes a novel intelligent node localization algorithm in WSN based on beacon nodes to improve the precision in location estimation. Firstly, system model of WSN node localization is constructed according to the WSN environment. Then traditional WSN node localization methods such as DV-HOP, GA, and PSO are studied. Localization algorithm of WSN is proposed by using dynamic mathematics modeling. And the result of simulation, which is compared to the traditional algorithm, indicated that this algorithm is better to improve the accuracy and coverage of WSN. The simulation results show that the performance of the proposed WSN location algorithm is better than the traditional localization algorithms.
\end{abstract}

\section{Introduction}

1.1. Background and Research Status. WSN has a great application future in the military and civil area. The fundamental problems of sensor networks are deployment and coverage, localization, and networking protocols [1-3]. The accuracy of node localization is crucial for many applications of distributed sensor network (DSN) [4-7]. The current node localization algorithm of WSN is mainly divided into two categories: one is a node location algorithm based on ranging and the other is a nonranging node localization algorithm. Since the distance-based node positioning algorithm requires additional auxiliary facilities, the resource consumption is relatively large. Therefore, it is not suitable for use in largescale networks. The nonranging node algorithm does not require additional ancillary facilities while hardware cost is relatively low. Therefore, it becomes a research hotspot in current wireless sensor networks. Our paper is based on the idea of a nonranging node algorithm. A new wireless sensor localization algorithm based on irregular node communication is proposed. The feasibility and effectiveness of the algorithm are verified by simulation experiments.

In general, positioning accuracy can be improved by increasing the number of anchor nodes. However, the cost of the anchor node is higher than that of ordinary nodes. If $10 \%$ of the nodes are anchor nodes, the price of the network will increase 10 times [8-13]. However, when the unknown node is located, it will no longer need expensive anchor nodes. Therefore, it is necessary to reduce the number of anchor nodes participating in node positioning. If the cost is reduced by reducing the number of anchor nodes, the consequent effect is a reduction in positioning accuracy. Based on the broadcast characteristics of wireless transmission, some methods based on geometric knowledge of interfering node positioning have been proposed, including centroid positioning, weight centroid positioning, iterative positioning 
of virtual forces, convex shell positioning, and alpha shell positioning. In the centroid localization method (CL, centroid localization), interfering nodes' neighboring nodes are called interfered nodes. The CL collects the coordinates of all disturbed nodes and uses the average as the estimated position of the interference node. Considering that different interfered nodes have different distances from interfering nodes, their perceived interference intensity is also different. The researchers proposed the positioning of weight centers of mass, which improved the positioning accuracy to some extent. Juan J. Galvez, Patrick Carroll,et al [14-20] proposed a novel probabilistic graphical model called Bayesian Network based Program Dependence Graph (BNPDG) that has the excellent inference capability across nonadjacent WSN nodes. They focused on applying the BNPDG at indoor node localization. Compared with the PPDG, their BNPDGbased localization approach overcomes the limitation across nonadjacent nodes and provides more precise localization by taking its output nodes as the common conditions to calculate the conditional probability of each nonoutput node. Yatish K.Joshi,Shi Zhang,et al [21-27] proposed a Heuristic Multidimensional Scaling (HMDS) algorithm to improve accuracy of node localization in anisotropic WSNs with holes. By exploring the virtual node and constructing the shortest paths between nodes, the Euclidean distances between nodes are obtained via employing the heuristic approach such that they can be used to calculate more accurate locations of the nodes. Ajinkya Rajandekar,et al [28, 29] investigated the significance of introducing small world characteristics in a conventional WSN for improving the node localization accuracy. A novel constrained iterative average path length reduction algorithm is proposed to introduce small world characteristics into a conventional WSN. The method utilizes a novel frequency selective approach for the introduction of small world phenomena.

The robust quadrilateral method for judging node rollover ambiguity is mostly applicable only to three-sided positioning using three reference nodes. Compared to the three-sided positioning method, multilateration methods using more than three reference nodes can get smaller average positioning errors. Therefore, in the node positioning of a wireless network based on ranging, a multilateration method is mostly used. At present, there is still very little research on the problem of node rollover ambiguity in multilateral positioning. Although some literatures have proposed a robust quadrilateral method suitable for multilateral positioning for the first time, however, its computational complexity is large and the judgment effect is poor.

Hao Zhang,Wei Liu, et al [30-32] proposed and proved that flip ambiguity detection for three-dimensional node localization is equivalent to the intersecting plane that intersects with all range error spheres of the reference nodes of unknown node in the ideal radio model, which is called the existence of intersecting plane (EIP) problem. They proposed common tangent plane algorithm (CTP) and orthogonal projection algorithm (OP). Hua-Dong Mo and Rodolfo Feick $[33,34]$ proposed a Bluetooth interior location algorithm based on Bayesian theory for RSSI probability distribution. The algorithm used the maximum posterior probability to determine the position of the moving target, and the algorithm is complicated. There is a large error. Min Jia and Limei Lin $[35,36]$ proposed an enhanced Gaussian mixture model WSN positioning algorithm; the algorithm achieved high positioning accuracy, but the algorithm is complex and difficult to implement on mobile terminals such as smart phones and wireless environment.

In the anchor node mobility strategy, there are many strategies in order to achieve certain coverage. They assume that the anchor node's moving path can cover the entire network, ignoring the actual distribution density of unknown nodes. The appearance of anchor nodes cannot effectively avoid traversing the network empty areas. It cannot provide good distribution of virtual beacons, long moving path, and low positioning accuracy because there is a close relationship between virtual force and node distribution. Literature [37] introduced the concept of virtual force potential field to the node coverage problem of wireless sensor networks for the first time. A wireless sensor network node coverage method based on virtual force potential field is proposed. Literature [38] has improved the above-mentioned virtual force potential field. A virtual force algorithm (VFA) has been proposed. The algorithm combined virtual gravity and virtual repulsion to determine the motion paths of randomly distributed nodes. Considering the energy consumption, the nodes will move uniformly after the sink node has calculated the final coordinates of each node.

1.2. Motivations. Above discussion implies that novel inequality methods allow us to obtain more precise localization and lower error. Most references do not consider the dynamic behaviors of wireless communication networks node. Under this method efficiency, novel node localization of WSN method and mathematics model could be proposed.

1.3. Our Contributions. The main contributions of this paper are listed as follows.

(1) System architecture of wireless sensor network is constructed. (2) A mesh generation method is constructed. (3) Wireless network node information exchange mechanism is established. (4) A new node localization algorithm is proposed.

1.4. Structure of the Paper. The rest of this paper is organized as follows. In Section 2, some traditional sensor node localization methods are introduced. In Section 3, system model of node localization of WSN is built according to the wireless communication network. In Section 4, a new node localization and dynamic modeling method is proposed. In Section 5, the simulation analytical results are presented. Finally, conclusions are drawn in Section 6.

\section{Sensor Node Localization Algorithm}

2.1. DV-HOP Node Localization Algorithms. The node localization algorithm of DV-Hop is a kind of distance vector method, while GPS is a distributed localization method. The algorithm can be divided into the following three steps [33, 34]. 
Step 1. Packet for each node is $\left(I D_{i}, x, y, h o p_{i}\right)$. The packet contains the coordinates information and the minimum hop. The initial value of the minimum hop is 0 .

Step 2. The first step is completed; all the beacon nodes know the minimum hop count from other beacon nodes. When a parameter HopSize is introduced, HopSize is named as the distance of average hop number.

Step 3. The distance between unknown node and beacon node is calculated by the minimum hop.

2.2. Genetic Algorithm (GA). In the GA sensor node localization algorithm, chromosomes are possible solutions for specific problems. Problems with parameters are called genes $[35,36]$. For 2-dimensional plane localization, each chromosome has two parameters, as $(x, y)$. Genetic algorithm has the following three basic operations: selection, crossover, and mutation. Through the cross, two parent individuals are combined to produce two new chromosomes; offspring inherited the characteristics of the parent. And the key is to create a new function of evolution; the genetic algorithm will need to make use of the mutation operator. A new generation is produced, followed by the fitness function of the evaluation. A new generation will be arranged from good to bad, until convergence or reaching the predetermined number of iterations.

2.3. Particle Swarm Optimization (PSO) Algorithm. The PSO $[37,38]$ sensor node localization algorithm consists of a number of moving particles; particles are responsible for finding the optimal solutions. The algorithm updates the particle's speed and location. Each particle goes through two extrema updating itself, namely, the particles themselves to find the optimal solution (local optimal) and the whole population to find the optimal solution (global optimal). The particle's velocity and location are updated according to some fixed rules. When convergence conditions of the algorithm are reached, the iterations are aborted.

\section{System Model}

3.1. System Architecture of Wireless Sensor Network. For environmental wireless node monitoring, wireless sensor network (WSN) consists of four parts. These four parts are the monitoring nodes (including the node and the route node), the main node (sink node), Internet (including satellite), and the upper computer, respectively, as can be seen from Figure 1.

In WSN, there is a system for node localization and environmental monitoring (such as Figure 1). Nodes usually have random distribution, and networks are in the form of self-organization. Monitoring nodes in WSN will sense and monitor the data directly or through the route node to anchor node (beacon node). Main nodes through Internet or satellite transmit to upper computers, which are installed as the receiving software upper computer receives this part of the data, with the object to be measured and the implementation of remote monitoring and real-time operation. In monitoring, nodes exist in the anchor or beacon nodes and unknown nodes, with the unknown node through its nearby beacon nodes to communicate. Using certain localization algorithm to draw their own position will eventually be its location information with other data sent to the upper computer, so that the smooth progress of monitoring and positioning can work.

3.2. Mesh Generation. The coverage area is meshed and the anchor node is at the mobile collection point. Cover the number through the ring logo corresponding grid values. As shown in Figure 2, the three subrings intersect in a certain area, and number 3 means that the mesh is surrounded by 3 rings covered; the actual location of the grid is covered by multiple rings; therefore, the higher number of covered grids is the more likely location for unknown nodes in the area. We can select the most consistent cluster unknown nodes; the best fitness grid and the coordinates of unknown node can be obtained by using node localization algorithm.

According to the node's location information and communication radius, the network area is divided into several virtual cells. In order to ensure that any two nodes in adjacent cells can communicate directly, suppose the node knows the location information of the whole monitoring area and its own location information, and the node can calculate which cell it belongs to. The principle of model construction, grid mesh generation, boundary condition definition algorithm, and governing equation solution are confirmed. The numerical examples showed that the method was efficient and accurate in solving the node grid generation of WSN by using finite coarse mesh and simple interpolating element.

It not only guarantees that any two nodes in the adjacent cells can communicate directly, but also can eliminate the effects of hidden terminals. The cell radius needs to meet

$$
(3 r)^{2}+(3 r)^{2} \leq R^{2} \Longrightarrow r \leq \frac{R}{3 \sqrt{2}}
$$

Therefore, from the perspective of packet forwarding, nodes belonging to the same cell can be considered equivalent, and each cell only needs to select one node to keep active. Moreover, it ensures that the neighboring cluster heads of a cluster head are in communication with each other. This ensures that the underlying MAC protocol can use the collision avoidance policy to prevent neighboring cluster heads from sending data simultaneously. As in Figure 3, such as cluster 5 , if $r>R / 3 \sqrt{2}$, then cluster heads 1 and 9 of cluster 5 can send data to cluster head 5 at the same time. In fact, in a sufficiently small time interval, it is not possible to send "application layer data" at the same time by two or more adjacent cluster heads.

\section{Sensor Node Localization Algorithm}

4.1. Dynamic Mathematics Modeling. In m-dimensional space, each sensor node can be measured with at least $\mathrm{m}$ nodes distance. In m-dimensional space, consider a set of sensor networks consisting of $\mathrm{N}$ sensor nodes. 

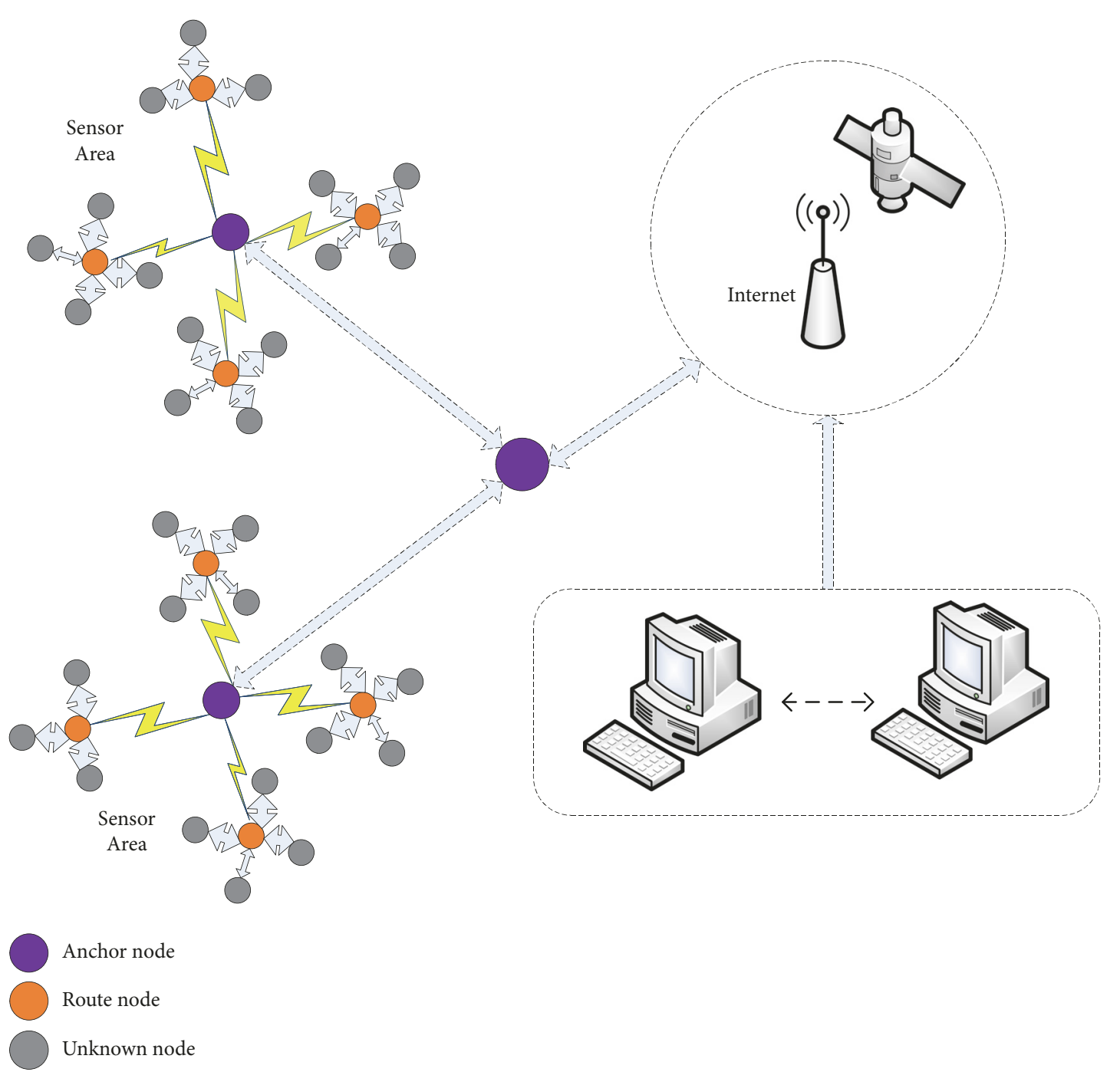

FIGURE 1: System architecture of wireless sensor network.

$\mathbf{K}=\{1,2, \ldots K\}$. The distance between node $i$ and node $j$ is $d_{i j}$. Distance matrix $\Delta(i, j)$ and connectivity relationship matrix $\mathbf{M}(i, j)$ can be defined as

$$
\begin{gathered}
\Delta(i, j)= \begin{cases}d_{i j} & j \neq i \\
0 & \text { esle }\end{cases} \\
\mathbf{M}(i, j)= \begin{cases}1 & j \neq i \\
0 & \text { esle }\end{cases}
\end{gathered}
$$

The motion model of a node can be expressed as

$$
\begin{aligned}
X_{i(t)} & =X_{i(t-1)}+\frac{1}{2} v_{i(t-1)} \times \Delta t\left[\begin{array}{c}
\cos \theta_{i(t-1)} \\
\sin \theta_{i(t-1)}
\end{array}\right] \\
v_{i(t)} & =\frac{1}{3} \omega \times v_{i(t-1)}+a \times \text { rand }
\end{aligned}
$$

where $\omega$ is inertial weight. $a$ is the acceleration factor. $r a n d$ is a random number between 0 and 1 .

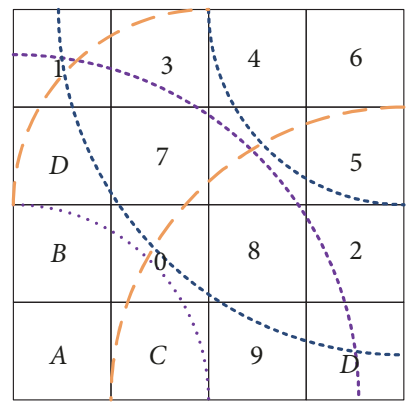

FIGURE 2: Mesh generation.

In the local coordinate of cluster $\mathbf{C}_{S}$, node $i \in \mathbf{C}_{S}$, the relative coordinate is $\mathbf{x}_{i}^{s}=\left[x_{i}^{s}, x_{i}^{s}, x_{i}^{s}\right]^{T}$. The relative coordinates of node $i$ can be calculated as

$$
\mathbf{x}_{i}^{0}=-\frac{1}{3}\left(A_{2}^{T} B^{T} B A_{2}\right)^{-1} A_{2}^{T} B^{T}
$$




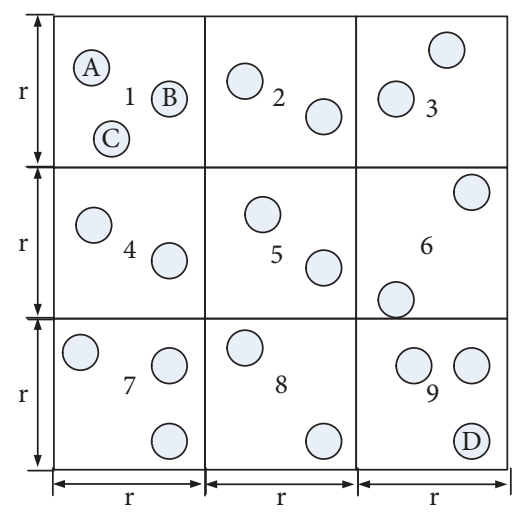

FIGURE 3: Virtual mesh division.

where $B=X X^{T}$ is the number of product matrixes. As for $\mathrm{M}$ nodes, $j, \ldots, q \in \mathbf{C}_{0} . A_{1}$ and $A_{2}$ are the first column of the matrix $\mathrm{A}$ and the remaining columns, respectively.

$$
A=\left[\begin{array}{c}
1_{M}^{T} \\
P^{T}
\end{array}\right]^{T}\left(\left[\begin{array}{c}
1_{M}^{T} \\
P^{T}
\end{array}\right]\left[\begin{array}{c}
1_{M}^{T} \\
P^{T}
\end{array}\right]^{T}\right)^{-1}
$$

Suppose the coordinates of unknown node 0 are $\left(x_{0}, y_{0}\right)$. Coordinates of beacon nodes $1,2,3,4$ are $\left(x_{1}, y_{1}\right),\left(x_{2}, y_{2}\right)$, $\left(x_{3}, y_{3}\right),\left(x_{4}, y_{4}\right)$. The distance from node 0 to 4 node $\mathrm{s}$ is, respectively,

$$
\begin{aligned}
& f_{1,0}=\rho_{1}-\left(\left(x_{1}-x_{0}\right)^{2}+\left(y_{1}-y_{0}\right)^{2}\right) \\
& f_{2,0}=\rho_{2}-\left(\left(x_{2}-x_{0}\right)^{2}+\left(y_{2}-y_{0}\right)^{2}\right) \\
& f_{3,0}=\rho_{3}-\left(\left(x_{3}-x_{0}\right)^{2}+\left(y_{3}-y_{0}\right)^{2}\right) \\
& f_{4,0}=\rho_{4}-\left(\left(x_{4}-x_{0}\right)^{2}+\left(y_{4}-y_{0}\right)^{2}\right)
\end{aligned}
$$

where $\rho_{i}$ indicates the learned distance from node 1 to node 0 . To solve the coordinate of node 0 , it makes $\sum_{i=1}^{4} f_{i, 0}$ minimum, as

$$
F\left(x_{0}, y_{0}\right)=\operatorname{Min}\left(f_{1,0}+f_{2,0}+f_{3,0}+f_{4,0}\right)
$$

Node relative localization and the radial error can be expressed, respectively, as

$$
\begin{aligned}
\text { error }_{\text {avg }}= & \frac{\sum_{i=1}^{n} \sqrt{\left(x_{i}-x_{i}^{\prime}\right)^{2}+\left(y_{i}-y_{i}^{\prime}\right)^{2}+\left(z_{i}-z_{i}^{\prime}\right)^{2}}}{n \times R} \\
& \times 100 \% \\
\text { error }_{x}= & \frac{\sum_{i=1}^{n} a b s\left(x_{i}-x_{i}^{\prime}\right)}{n \times R} \times 100 \%
\end{aligned}
$$

where $n$ is the number of sensor nodes and $R$ is the wireless communication radius.

Suppose the coordinates of the unknown node are $(x, y)$ and the coordinates of the obtained beacon node and the corresponding distance to the beacon node are $\left(x_{1}, y_{1}, d_{1}\right),\left(x_{2}, y_{2}, d_{2}\right) \ldots\left(x_{n}, y_{n}, d_{n}\right)$. Some formulas can be obtained according to the two-dimensional plane distance. Taylor decomposition of formula (13) at point $\left(x_{0}, y_{0}\right)$ can be described as

$$
\begin{aligned}
f(x, y)= & f\left(x_{0}+h, y_{0}+h\right) \\
= & f\left(x_{0}, y_{0}\right)+\left(h \frac{\partial}{\partial x}+k \frac{\partial}{\partial y}\right) f\left(x_{0}, y_{0}\right) \\
& +\frac{1}{2 !}\left(h \frac{\partial}{\partial x}+k \frac{\partial}{\partial y}\right)^{2} f\left(x_{0}, y_{0}\right)+\cdots
\end{aligned}
$$

When $(h, k)$ is very small, formula (13) can be simplified as

$$
\begin{aligned}
f(x, y)= & f\left(x_{0}+h, y_{0}+h\right) \\
= & \sqrt{\left(x_{0}-x_{n}\right)^{2}+\left(y_{0}-y_{n}\right)^{2}} \\
& +\frac{\left(x_{0}-x_{n}\right)}{\sqrt{\left(x_{0}-x_{n}\right)^{2}+\left(y_{0}-y_{n}\right)^{2}}} h \\
& +\frac{\left(y_{0}-y_{n}\right)}{\sqrt{\left(x_{0}-x_{n}\right)^{2}+\left(y_{0}-y_{n}\right)^{2}}} k
\end{aligned}
$$

\subsection{The Flow Of Algorithm.}

Step 1. For each node $i \in \mathbf{K}$, search for all nodes that it can measure and construct the set $\mathbf{S}_{i}=\{j \in \mathbf{K} \mid M(i, j)=1\}$.

Step 2. For each node $i \in \mathbf{K}$, update the matrix $\mathbf{S}_{t}=\mathbf{S}_{i} \cup$ $\mathbf{S}_{j}, \forall j \in \mathbf{S}_{i}$.

Step 3. For all nodes in the sensor network, search for the maximum potential set, recorded as $\mathbf{S}_{t}^{*}$. All nodes in $\mathbf{S}_{t}^{*}$ make reference cluster $\mathbf{C}_{0}$, and update the set $\mathbf{K}_{0}=\mathbf{K} / \mathbf{S}_{t}^{*}$.

Step 4. For all nodes in set $\mathbf{K}_{0}$, search for the maximum potential set, recorded as $\mathbf{S}_{l}^{*}$. All nodes in $\mathbf{S}_{t}^{*}$ make cluster $\mathbf{C}_{S}$, $S=1,2, \ldots$. Then repeat this step until $\mathbf{K}_{0}=\varnothing$.

Step 5. As for each cluster $\mathbf{C}_{S}$, calculate the relative coordinates of nodes in a cluster, and construct a local coordinate map of each cluster.

\section{Simulation Analysis}

In $150 m \times 150 m$ conventional network environment(referred to as square-random), randomly place 400 wireless sensor nodes. In the sensor area, the radiation distance of each broadcast can reach the whole network. The communication model of the node defaults to square-random model, and the beacon node and the unknown node have the same communication radius.

It can be seen from Figure 4 that as density of beacon node is increasing, the error of localization is decreasing. In the case of the same beacon node density, the proposed method is better than the several traditional sensor node localization methods, such as DV-HOP, GA, and PSO. When 


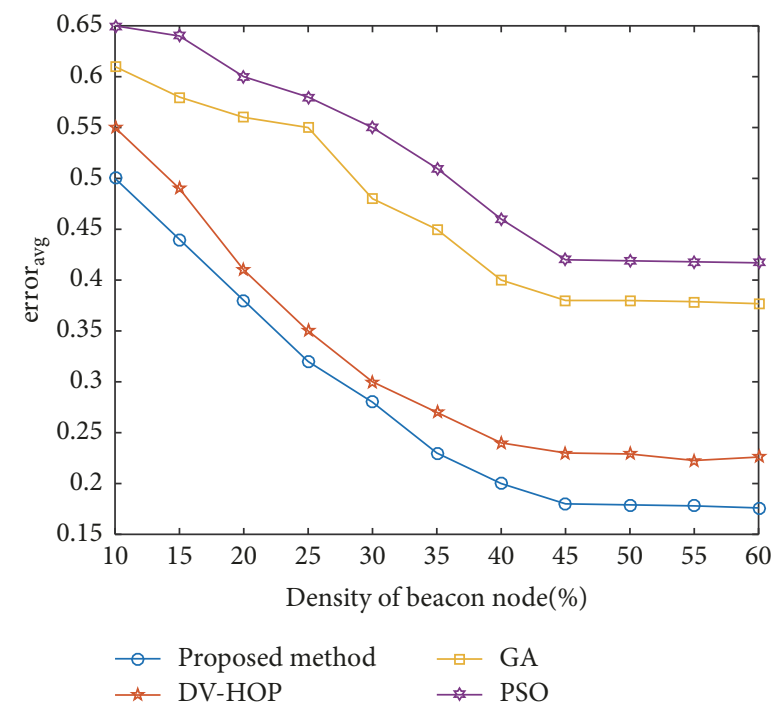

FIGURE 4: Relation between beacon node density and location error.

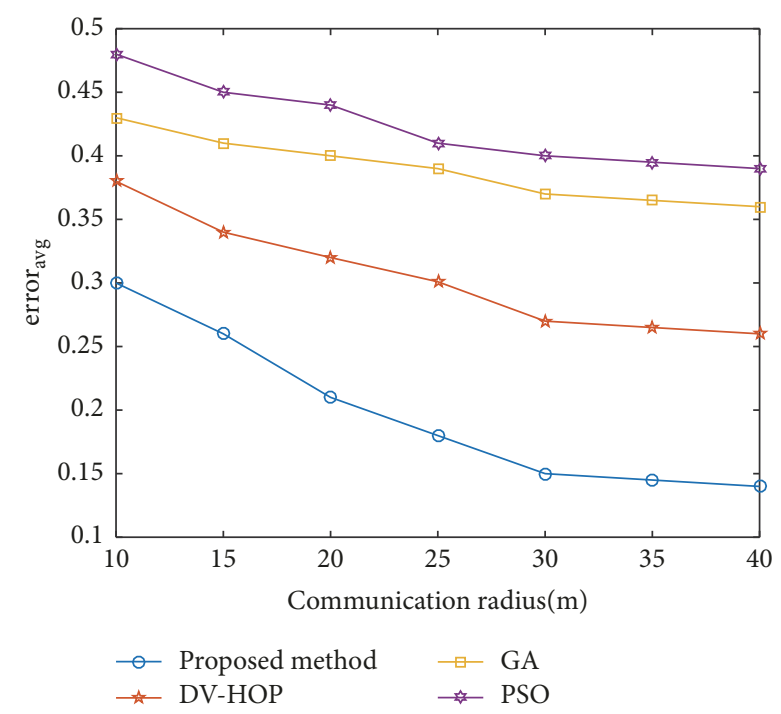

FIGURE 5: Relation between node communication radius and location error.

the density of beacon node is about $10 \%$, the localization accuracy of proposed method is not obvious compared with the classical algorithms. Because the proportion of beacon nodes is relatively small, the beacon nodes located near unknown nodes are also relatively small. The proportion of beacon nodes is increasing. Accordingly, the minimum number of hops between unknown nodes and beacon nodes is also reduced, so that the distance between them is closer to the true value, and the localization error is also effectively reduced. When the proportion of beacon nodes is increased to about $45 \%$, the localization error is basically stable, but localization accuracy of proposed method is much more improved.

As can be seen from Figure 5, we consider the relationship between node communication radius and error. With node communication radius increasing, the error of localization

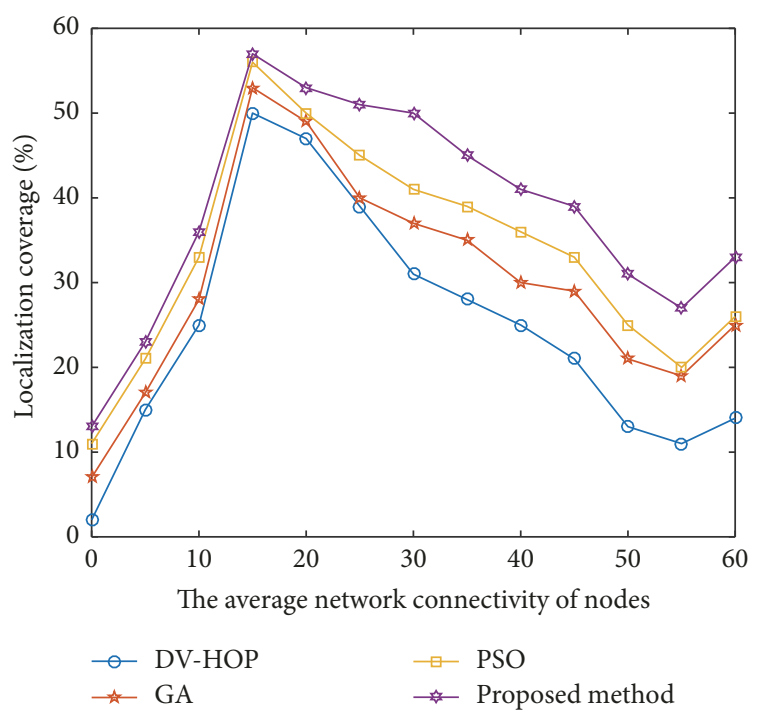

FIGURE 6: Coverage changes with network connectivity.

is decreasing. In the case of the same node communication radius, the proposed method is better than DV-HOP,GA, and PSO sensor node localization methods. However, when the node communication distance increases to a certain extent (such as $30 \mathrm{~m}$ ), the node localization accuracy of the algorithms does not increase or decrease much.

Due to the cost and power consumption of sensor nodes, the smaller the network connectivity (Node Density, ND) and the density of beacon node (Anchor Density, AD), the better the system performance in WSN.

As can be seen from Figure 6, with the increase in connectivity of nodes, the localization coverage is increased, which has a clear effect of WSN node localization. When the value of average network connectivity nodes reached about 18 , the localization coverage is becoming reduced. When the value of localization coverage reached nearly 56, the localization coverage has shown a rising trend recently.

As can be seen from Figure 6 and Figure 7, proposed method's location coverage advantage over DV-HOP,GA, and $\mathrm{PSO}$ is more pronounced when ND or AD is smaller because the neighbor discovery algorithm allows the node to obtain more beacon node information, so that they can complete the positioning process.

\section{Summary}

A new WSN localization algorithm is proposed in this paper by using dynamic modeling method. Traditional sensor node localization algorithms such as PSO, GA, and DV-HOP are studied. System architecture of WSN is constructed according to the WSN localization environment, and then the method of mesh generation is researched. Results show that the performance of the proposed location algorithm is better than the traditional methods.

The future direction of the research is as follows:

(a) Energy efficiency of WSN node deployment. 


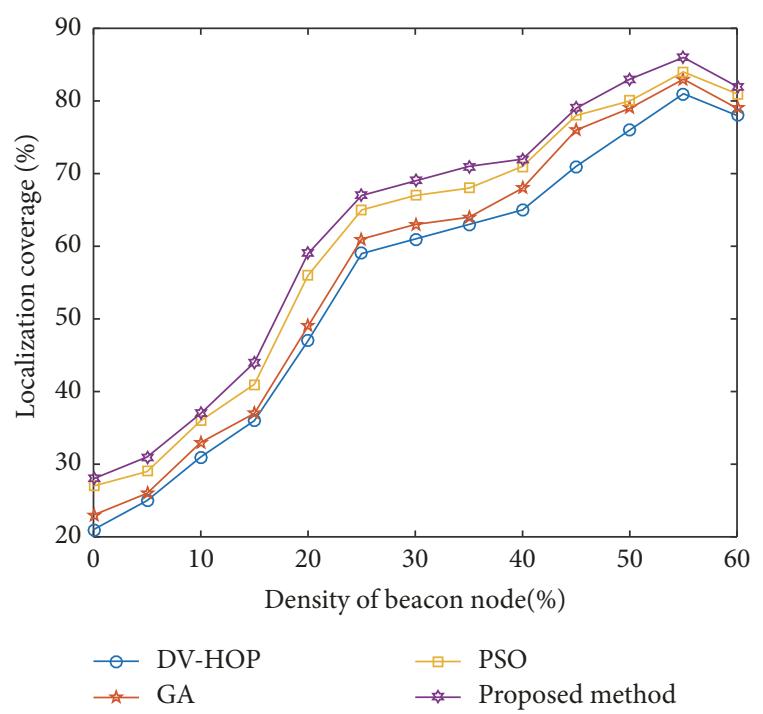

FIGURE 7: Coverage changes with beacon node density.

(b) Key distribution protocol is one of significant components for security defense on WSN transmission.

(c) Reducing the source consumption and prolonging the WSN lifecycle are becoming the chief target of WSN, which was based on the assurance of network secure communication.

\section{Conflicts of Interest}

The authors declare that there are no conflicts of interest regarding the publication of this paper.

\section{Acknowledgments}

The paper was supported by the Science and Technology Research Program of Chongqing Municipal Education Commission (KJ1600923, KJ17092060), Humanities and Social Sciences Research of Ministry of Education of China (16YJC860010), National Social Science Fund of China West Project (17XXW004), Social Science of Humanity of Chongqing Municipal Education Commission (17SKG144), Natural Science Foundation of China $(61571069,61501065$, 61502064, 61503052). The author Xiaoyang Liu acknowledges the financial support from CSC (China Scholarship Council) (no. 201608505142).

\section{References}

[1] W. Jiang, C. Gu, and J. Wu, "A Dynamically Reconfigurable Wireless Sensor Network Testbed for Multiple Routing Protocols," Wireless Communications and Mobile Computing, vol. 2017, Article ID 1594270, 10 pages, 2017.

[2] A. Fichera, M. Frasca, and R. Volpe, "Complex networks for the integration of distributed energy systems in urban areas," Applied Energy, vol. 193, pp. 336-345, 2017.
[3] I. S. Baruch, V. A. Quintana, and E. P. Reynaud, "Complexvalued neural network topology and learning applied for identification and control of nonlinear systems," Neurocomputing, vol. 233, pp. 104-115, 2017.

[4] Y. Wang, L. Bi, S. Lin, M. Li, and H. Shi, "A complex networkbased importance measure for mechatronics systems," Physica A: Statistical Mechanics and its Applications, vol. 466, pp. 180198, 2017.

[5] L. E. C. Rocha, "Dynamics of air transport networks: A review from a complex systems perspective," Chinese Journal of Aeronautics, vol. 30, no. 2, pp. 469-478, 2017.

[6] A. Xenakis, F. Foukalas, and G. Stamoulis, "Cross-layer energyaware topology control through Simulated Annealing for WSNs," Computers and Electrical Engineering, vol. 56, pp. 576590, 2016.

[7] G. Chen, B. Chen, P. Li et al., "Study of aerodynamic configuration design and wind tunnel test for solar powered buoyancylifting vehicle in the near-space," Procedia Engineering, vol. 99, pp. 67-72, 2015.

[8] H. Liao, A. Zeng, M. Zhou, R. Mao, and B. Wang, "Information mining in weighted complex networks with nonlinear rating projection," Communications in Nonlinear Science and Numerical Simulation, vol. 51, pp. 115-123, 2017.

[9] B. Cai, H. Liu, and M. Xie, "A real-time fault diagnosis methodology of complex systems using object-oriented Bayesian networks," Mechanical Systems and Signal Processing, vol. 80, pp. 31-44, 2016.

[10] X. Desforges, M. Diévart, and B. Archimède, "A prognostic function for complex systems to support production and maintenance co-operative planning based on an extension of object oriented Bayesian networks," Computers in Industry, vol. 86, pp. 34-51, 2017.

[11] J. Fan, Z. Wang, and G. Jiang, "Quasi-synchronization of heterogeneous complex networks with switching sequentially disconnected topology," Neurocomputing, vol. 237, pp. 342-349, 2017.

[12] X. Liu, X. Zeng, C. Liu, W. Liu, and Y. Zhang, "The channel estimation and modeling in high altitude platform station wireless communication dynamic network," Discrete Dynamics in Nature and Society, vol. 2017, Article ID 5939810, 7 pages, 2017.

[13] N. Baccour, A. Koubâa, H. Youssef, and M. Alves, "Reliable link quality estimation in low-power wireless networks and its impact on tree-routing," Ad Hoc Networks, vol. 27, pp. 1-25, 2015.

[14] J. J. Gálvez and P. M. Ruiz, "Joint link rate allocation, routing and channel assignment in multi-rate multi-channel wireless networks," Ad Hoc Networks, vol. 29, article no. 1198, pp. 78-98, 2015.

[15] H. Dai, W. Chen, J. Jia, J. Liu, and Z. Zhang, "Exponential synchronization of complex dynamical networks with timevarying inner coupling via event-triggered communication," Neurocomputing, vol. 245, pp. 124-132, 2017.

[16] J. Jian and P. Wan, "Lagrange $\alpha$-exponential stability and $\alpha$ exponential convergence for fractional-order complex-valued neural networks," Neural Networks, vol. 91, pp. 1-10, 2017.

[17] R. Rakkiyappan, G. Velmurugan, J. N. George, and R. Selvamani, "Exponential synchronization of Lur'e complex dynamical networks with uncertain inner coupling and pinning impulsive control," Applied Mathematics and Computation, vol. 307, pp. 217-231, 2017.

[18] B. Varghese, N. E. John, S. Sreelal, and K. Gopal, "Design and Development of an RF Energy Harvesting Wireless Sensor 
Node (EH-WSN) for Aerospace Applications," in Proceedings of the 6th International Conference On Advances In Computing and Communications, ICACC 2016, pp. 230-237, September 2016.

[19] J. Lunze, "Structural properties of networked systems with random communication links," Automatica, vol. 80, pp. 300304, 2017.

[20] X. Liu, C. Liu, and W. Liu, "Wind shear target echo modeling and simulation," Discrete Dynamics in Nature and Society, vol. 2015, Article ID 479804, 6 pages, 2015.

[21] Y. K. Joshi and M. Younis, "Restoring connectivity in a resource constrained WSN," Journal of Network and Computer Applications, vol. 66, pp. 151-165, 2016.

[22] L. A. Bakhtiar, M. Hosseinzadeh, and M. Reshadi, "Reliable communications in optical network-on-chip by use of fault tolerance approaches," Optik - International Journal for Light and Electron Optics, vol. 137, pp. 186-194, 2017.

[23] H. Mosbah and M. E. El-Hawary, "Optimization of neural network parameters by Stochastic Fractal Search for dynamic state estimation under communication failure," Electric Power Systems Research, vol. 147, pp. 288-301, 2017.

[24] I. Jawhar, N. Mohamed, J. Al-Jaroodi, D. P. Agrawal, and S. Zhang, "Communication and networking of UAV-based systems: Classification and associated architectures," Journal of Network and Computer Applications, vol. 84, pp. 93-108, 2017.

[25] C. Peng, E. Tian, J. Zhang, and D. Du, "Decentralized eventtriggering communication scheme for large-scale systems under network environments," Information Sciences, vol. 380, pp. 132-144, 2017.

[26] A. Morelli, M. Tortonesi, C. Stefanelli, and N. Suri, "Information-Centric Networking in next-generation communications scenarios," Journal of Network and Computer Applications, vol. 80, pp. 232-250, 2017.

[27] Y. Yan, B. Zhang, and C. Li, "Opportunistic network coding based cooperative retransmissions in D2D communications," Computer Networks, vol. 113, pp. 72-83, 2017.

[28] A. Rajandekar and B. Sikdar, "A survey of MAC layer issues and protocols for machine-to-machine communications," IEEE Internet of Things Journal, vol. 2, no. 2, pp. 175-186, 2015.

[29] W. Ahmed, O. Hasan, U. Pervez, and J. Qadir, "Reliability modeling and analysis of communication networks," Journal of Network and Computer Applications, vol. 78, pp. 191-215, 2017.

[30] H. Zhang and G. Dai, "Design of optical fiber communication network monitoring and detection system based on address resolution protocol cheats," Optik - International Journal for Light and Electron Optics, vol. 127, no. 23, pp. 11242-11249, 2016.

[31] M. Aslam, E. U. Munir, M. M. Rafique, and X. Hu, "Adaptive energy-efficient clustering path planning routing protocols for heterogeneous wireless sensor networks," Sustainable Computing, vol. 12, pp. 57-71, 2016.

[32] J. Wu, Z.-J. Zhou, X.-S. Zhan, H.-C. Yan, and M.-F. Ge, "Optimal modified tracking performance for MIMO networked control systems with communication constraints," ISA Transactions ${ }^{\circledR}$, vol. 68, pp. 14-21, 2017.

[33] H.-D. Mo, Y.-F. Li, and E. Zio, "A system-of-systems framework for the reliability analysis of distributed generation systems accounting for the impact of degraded communication networks," Applied Energy, vol. 183, pp. 805-822, 2016.

[34] S. Sharma, Y. Shi, Y. Thomas Hou, S. Kompella, and S. F. Midkiff, "Network-coded cooperative communications with multiple relay nodes: Achievable rate and network optimization," Ad Hoc Networks, vol. 53, pp. 79-93, 2016.
[35] R. Feick, M. Rodriguez, L. Ahumada, R. A. Valenzuela, M. Derpich, and O. Bahamonde, "Achievable Gains of Directional Antennas in Outdoor-Indoor Propagation Environments," IEEE Transactions on Wireless Communications, vol. 14, no. 3, pp. 1447-1456, 2015.

[36] M. Jia, X. Liu, Z. Yin, Q. Guo, and X. Gu, "Joint cooperative spectrum sensing and spectrum opportunity for satellite cluster communication networks," Ad Hoc Networks, vol. 58, pp. 231238,2017

[37] Z. Xu, D. Zhang, and H. Song, "Distributed synchronization control of complex networks with communication constraints," ISA Transactions ${ }^{\circledR}$, vol. 65, pp. 186-198, 2016.

[38] L. Lin, L. Xu, S. Zhou, and Y. Xiang, "Trustworthinesshypercube-based reliable communication in mobile social networks," Information Sciences, vol. 369, pp. 34-50, 2016. 


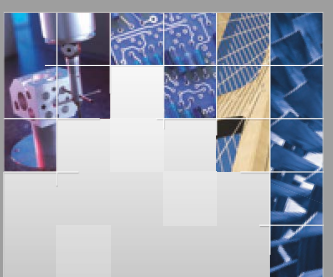

\section{Enfincering}
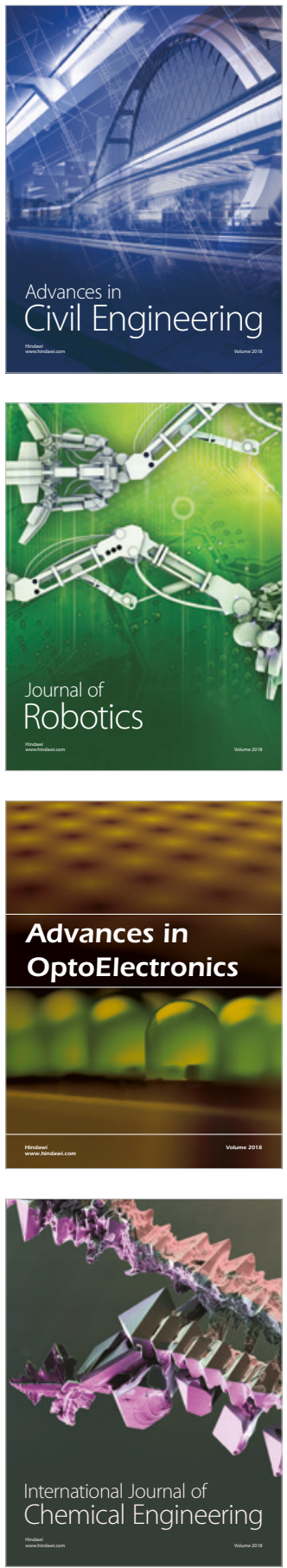

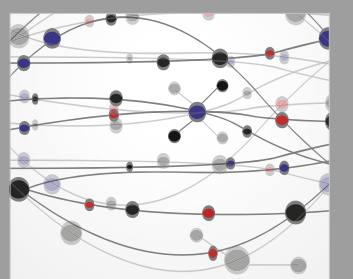

\section{Rotating \\ Machinery}

The Scientific World Journal

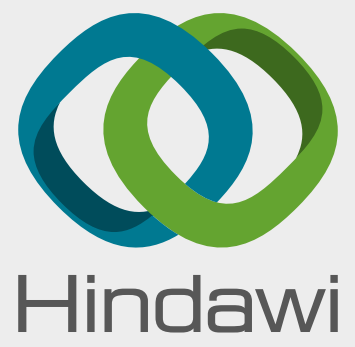

Submit your manuscripts at

www.hindawi.com
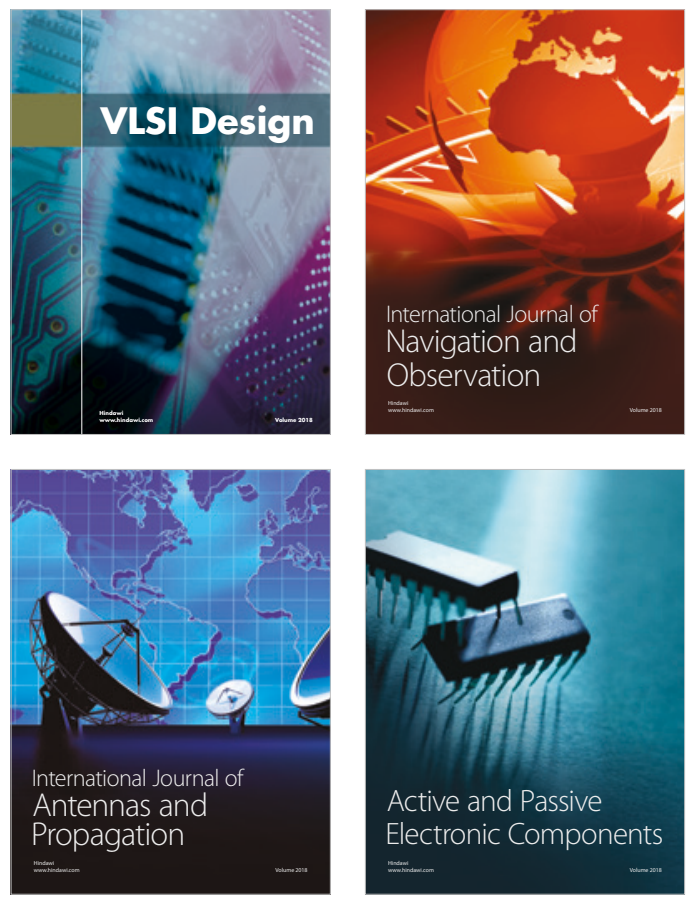
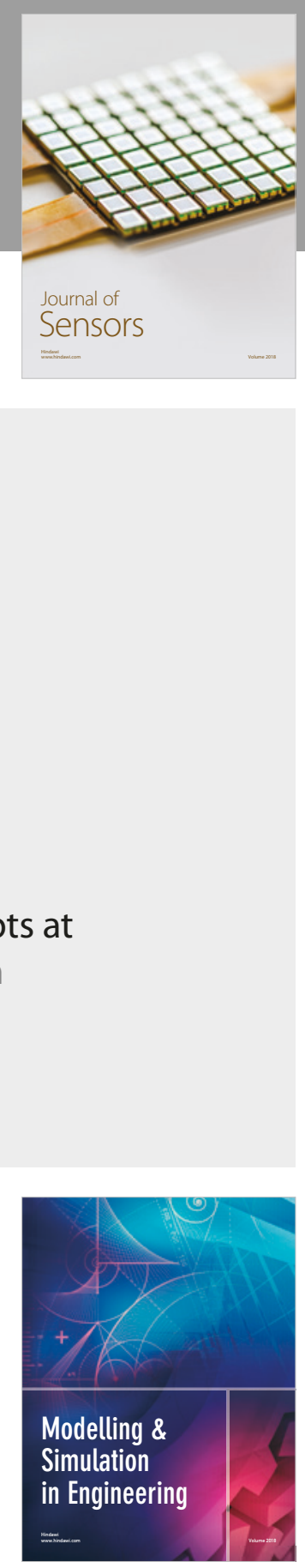

\section{Advances \\ Multimedia}
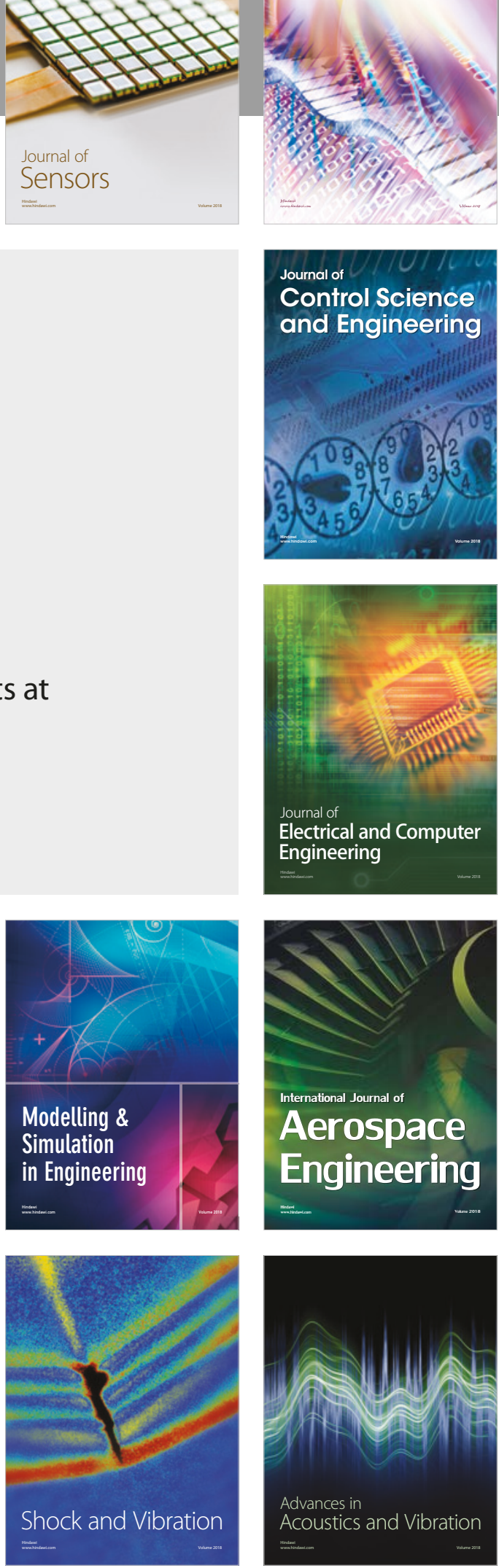